\title{
Manifestations and Treatment of Adult-onset Symptomatic Optic Pathway Glioma in Neurofibromatosis Type 1
}

\author{
JULIANE MEHLAN ${ }^{1}$, FRANK SCHÜTTAUF ${ }^{1}$, JOHANNES M. SALAMON ${ }^{2}$, \\ UWE KORDES $^{3}$, REINHARD E. FRIEDRICH ${ }^{4}$ and VICTOR-FELIX MAUTNER ${ }^{5}$ \\ ${ }^{1}$ Clinic of Ophthalmology, Medical Center Hamburg-Eppendorf (UKE), Hamburg, Germany; \\ ${ }^{2}$ Department for Diagnostic and Interventional Radiology and Nuclear Medicine, \\ University Medical Center Hamburg-Eppendorf, Hamburg, Germany; \\ ${ }^{3}$ Department of Pediatric Hematology and Oncology, \\ University Medical Center Hamburg-Eppendorf, Hamburg, Germany; \\ ${ }^{4}$ Department of Oral and Craniomaxillofacial Surgery, \\ University Medical Center Hamburg-Eppendorf, Hamburg, Germany; \\ ${ }^{5}$ Department of Neurology, University Medical Center Hamburg-Eppendorf, Hamburg, Germany
}

\begin{abstract}
This report describes the diagnosis and treatment of a 27-year-old patient with neurofibromatosis 1 (NF1) and late progression of a pre-existing optic pathway glioma $(O P G)$ that caused significant reduction in vision. $O P G$ is one of the diagnostic criteria for establishing the diagnosis of NF1. Most common findings of NF1 are café-au-lait spots, axillary and inguinal freckling of the skin, iris hamartoma (Lisch nodules), and tumors of the central nervous system and peripheral nerves. We successfully applied a modified International Society of Paediatric Oncology chemotherapy regimen for low-grade glioma in children with carboplatin dose adjustment according to the area under the plasma drug concentration-time curve calculation. During and after the chemotherapy, a clear improvement of the visual capacity was achieved. Age-adapted chemotherapy for symptomatic adult-onset OPG in patients with NF1 should be considered in individual cases.
\end{abstract}

Neurofibromatosis type 1 (NF1) is the most common neurocutaneous disorder. Recklinghausen's neurofibromatosis is the earlier eponymous designation of the disease, von Recklinghausen being the first to provide a complete description of the histological components of this entity (1). $\mathrm{NF} 1$ prevalence is about one in 3,000 (2). The disease occurs in all ethnic groups (3). The NFI gene was identified on

Correspondence to: J. Mehlan, MD, Department of Ophthalmology, University Clinic Hamburg-Eppendorf, 52 Martinistrasse, 20246 Hamburg, Germany. Tel: +49 040741018812, e-mail: j.mehlan@uke.de

Key Words: Optic pathway glioma, neurofibromatosis type 1, vision, chemotherapy. chromosome 17 (3). Inheritance is autosomal dominant with complete penetrance. However, the phenotype varies considerably. Several key features of the disease affect the integument, such as café-au-lait spots, axillary and inguinal freckling, and iris hamartoma (Lisch nodules). From the fifth year of life, $60-70 \%$ of children with NF1 exhibit choroidal abnormalities (4). However, hallmarks of the disease are tumors of peripheral nerves. Plexiform neurofibroma is a characteristic tumor of $\mathrm{NF} 1$, which may also affect the orbit (5) and is in principle considered precancerous (2). Despite the preferential manifestation of tumors in the peripheral nervous system, the former term 'peripheral neurofibromatosis' is obsolete because in NF1, relatively large numbers of patients may experience tumors of the central nervous system (2).

Central nervous system tumors in NF1. Pilocytic astrocytoma (PA) is the most common tumor of the central nervous system in patients with NF1 (2). Hudson was the first investigator to describe optic pathway glioma (OPG) as a distinct entity that is clearly distinguishable from other PAs (5-7). OPG may arise in the optic nerve but can also develop anywhere along the optic tract (2). Later, the connection between OPG and neurofibromatosis was revealed (6-8). Recent studies indicate a preference for the female sex (911 ), but this imbalance is not yet sufficiently proven (12). King et al. showed that Caucasian children have a much higher prevalence of OPG than children of African American descent (13). However, these ethnic differences are no longer emphasized (14).

The prevalence of OPG in patients with NF1, primarily children, is reported in the literature to be between 9.5 and $25 \%$ (15-18). Current estimates of the prevalence of OPG in NF1 suggest that approximately one in five patients develop this 
neoplasm (15). The occurrence of OPG in patients with NF1 is in the vast majority of cases in childhood. In two studies, the mean age at the time of diagnosis was 4.2 years and 5.8 years, respectively $(10,19)$. In general, the age of manifestation is mostly up to the age of six years, rarely after. The median age at diagnosis for NF1-associated OPG is later than non NF1associated OPG, however. There are only a few isolated reports, which describe later manifestations in NF1-affected individuals. A study by Lewis et al. in 1984 showed that the mean age at diagnosis in the population they observed was 15 years (20). However, this study was performed prior to establishment of National Institute of Health diagnostic criteria on NF1 (2). On the other hand, a more recent study reported the mean age of manifestation was 12.2 years (21). These differences in the age structure of the first diagnosed patients in several studies may be explained both by increased awareness as well as by local diagnostic capacities. Common to all studies is the finding that OPG is a diagnosis of early life in patients with NF1.

If OPG becomes symptomatic, then in the overwhelming majority, the tumor is already symptomatic during this phase of life. However, many tumors remain asymptomatic and require no therapy. Estimates foresee an asymptomatic course in $50 \%$ of cases in NF1-affected individuals. OPG can lead to vision loss and proptosis; Dodge stage II or III disease may cause endocrine disturbances such as precocious puberty. Typical diencephalic dystrophy is more common in non-NF1 OPG. The treatment of OPG occurring in those of young age often proves to be most difficult. Treatment philosophy of OPG has changed dramatically in recent decades and was significantly influenced by the drug therapy during childhood.

Chemotherapy. Most non-NF1 PAs harbor a single mutation of the proto-oncogene BRAF leading to BRAF/mitogenactivated protein kinase (MAPK) pathway activation. Similarily in NF1-associated PAs, loss of neurofibromin activates the RAS/RAF/MAPK pathway, thus inhibition of this pathway is being evaluated in current clinical trials.

At present, conventional chemotherapy is the standard measure for inoperable symptomatic or progressive PA both in pediatric NF1 and non-NF1. The frontline non-surgical approach constitutes a combined application of carboplatin and vincristine as established by the International Society of Pediatrics (SIOP) and Children's Oncology Group. The SIOP regimen includes an induction phase of 21 weeks with application of carboplatin $\times 7$ and vincristine $\times 13$, followed by a consolidation phase with cycles of carboplatin every 6 weeks and vincristine (days 1, 7 and 14). The objective response rate to this sequential therapy was higher in NF1associated OPG compared to non-NF1 OPG. Thus, the NF1 cohort was exempted from randomization in the follow-up trials, i.e. combined therapy with thioguanine, procarbazine, 1-(2-chloroethyl)-3-cyclohexyl-1-nitrosourea (CCNU) and vincristine vs. standard in Children's Oncology Group (carboplatin and vincristine, or thioguanine, procarabzine, CCNU and vincristine) or intensified induction (carboplatin/ vincristine/etoposide) vs. standard SIOP therapy (22).

Cumulative vincristine exposure is high following the SIOP regimen, with well-known side-effects particularly in older patients. Carboplatin allergy occurs in a sizable proportion of patients with a need to switch to alternative consolidation depending on the exact regimen given. Weekly vinblastine monotherapy was introduced by the Toronto group (23) and is already being used as a frontline standard by many centers. It will also be one of three randomized arms in the planned LOGGIC trial for standardized visual assessment and treatment indications including children with visual pathway glioma (24), next to the other experimental arm with a MAPK/ERK kinase inhibitor, and to standard carboplatin/vincristine.

Radiotherapy $(R T)$. In most circumstances $\mathrm{RT}$ is reserved for subsequent progression after failure of prior systemic therapies. Focal irradiation offers effective second-line nonsurgical management in OPG and a wide variety of techniques have evolved. Fractionated highly conformal stereotactic irradiation with photons is being utilized (27), as is particle therapy and radiosurgical approaches such as brachytherapy. All techniques attempt to spare exposure to surrounding risk organs. The advantage of proton therapy is the ability to apply a high dose of radiation to a localized site. The energy drop rate is very rapid, so that surrounding tissue can be spared (25). Depending on location and extent of disease, the accepted age for primary RT has shifted from 8 to beyond 16 years in the SIOP treatment for non-NF1 OPG. In general, irradiation should be avoided in the NF1 cohort $(26,27)$ because of an increased risk for radiogenic vasculopathy, malignant transformation and neurocognitive complications.

Surgery. Surgical procedures for OPG may be required for immediate debulking and clearance of cerebrospinal fluid obstruction in select cases. Excision of the affected optic nerve to stop progress via the chiasm, previously a common approach, is controversial, as retrograde progression of localized anterior disease in non-NF1 OPG is practically not observed (26). Biopsy, when feasible with justifiable operative risk, is only used when clinical images are not characteristic (27). In contrast to pediatrics, the risk group stratification and therapy recommendations for the much rarer adult PAs are less well defined. Small series suggest that resection is best followed by close observation, reserving RT for salvage or for biopsy-only patients (28).

Efficacy of adjuvant chemotherapy has been demonstrated for some adult non-pilocytic low-grade glioma. RTOG 9082 improved outcome with addition of procarbazine, $\mathrm{CCNU}$ and 


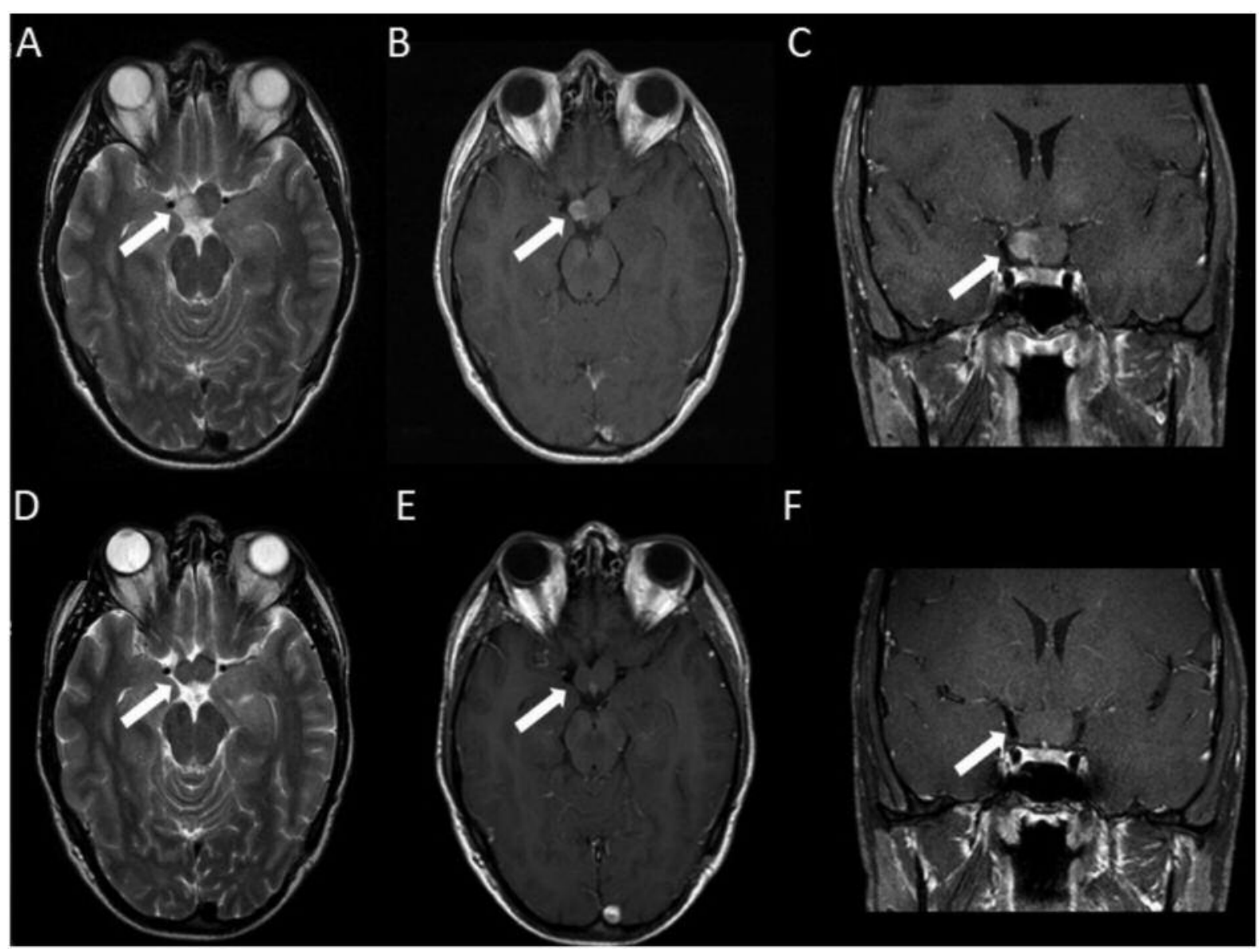

Figure 1. Upper row: Magnetic resonance imaging (MRI) before treatment. A: Axial T2 turbo spin-echo (TSE) sequences showing edema (white arrow) in the chiasma and right proximal optic nerve, as well as tumorous distension of both proximal optic nerves. B: Axial and C: coronal contrastenhanced T1 TSE MRI showing disturbances of the blood-brain barrier (white arrow) predominantly localized in the chiasma and right proximal optic nerve, with considerable displacement of the pituitary stem. Bottom row: MRI after treatment. D: Axial T2 TSE MRI showing complete decrease of edema (white arrow) and a clear decrease of the space-occupying mass. E: Axial and F: coronal contrast-enhanced T1 TSE MRI showing complete remission of the blood-brain barrier disturbances (white arrow) and no more displacement of the pituitary stem.

vincristine to RT (29) for high-risk adult low-grade glioma, as did a study from University of San Francisco with postoperative temozolomide (30), and more recently, early results from EORTC 22033-26033 showed equivalent progressionfree survival in molecularly defined adult low-grade glioma subgroups randomized between dose-dense temozolomide or RT (31). Carboplatin/vincristine experience in adult PA is not on record as far as we are aware.

\section{Case Report}

We report the case of a 27-year-old patient with NF1 who was treated with chemotherapy for first symptomatic progression of an optic glioma at the age of 21 years (in 2012). Development delay was present with 1 year, and subsequently café-au-lait spots, axillary and inguinal freckling led to clinical diagnosis of NF1. Furthermore, delayed motor skills, and dysgrammatism but no other clinical abnormalities were found. Regular magnetic resonance imaging (MRI) surveillance showed focal areas of signal intensity in the globus pallidus. Thickening of the left optic chiasm, the left-sided optic tract and the optic nerve were seen on MRI at the age of 15 years. At that time, full visual acuity was preserved, with only some enlargement of the blind spot in the visual field examination. Minimal progression of the OPG was first detected on MRI in 2011. Three years later, further progression was observed, including the right side, in particular the pre-chiasmatic part (Figure 1). Visual acuity of the right (0.5) and the left (0.63) eye was reduced; temporal visual field defects (right: diffuse scotoma, left: absolute scotoma) and reduced visually evoked 
Table I. Overview of the chemotherapeutical doses between 6/2014 and 12/2015.

Time period

Induction phase
$25 / 06 / 2014$ and $26 / 06 / 2014$

$17 / 07 / 2014$ and $18 / 07 / 2014$

$11 / 09 / 2014$ and $12 / 09 / 2014$

$08 / 10 / 2014$ and $09 / 10 / 2014$

$03 / 11 / 2014$ and $04 / 11 / 2014$

December 2014

Consolidation phase

01/2015-12/2015
Carboplatin $(+2 \mathrm{mg}$ vincristine $)$

$830 \mathrm{mg}$

$815 \mathrm{mg}$

$830 \mathrm{mg}$

$830 \mathrm{mg}$

$790 \mathrm{mg}$

$650 \mathrm{mg}$

$650 \mathrm{mg}$ potentials were diagnosed. These findings prompted the indication for adjuvant chemotherapy (Table I). From June 2014 until December 2014, SIOP induction chemotherapy for low-grade glioma was given modified with carboplatin dose adjusted according to the area under the curve (AUC; $830 \mathrm{mg}$ single dose; dose equivalent to AUC5) and vincristine $(2.0 \mathrm{mg}$ single dose). Visual acuity increased to 1.0 on the right and 0.8 on the left in 2015. Continuous regression throughout treatment was observed on subsequent MRI. The patient's visual acuity stabilized. Recovery of the field of vision could not be detected with certainty, however. Following the end of treatment in January 2016, stable disease was seen (Figure 1). Initially therapy was well tolerated, but after week 19, the carboplatin dose was reduced because of nausea and vomiting. Myelotoxicity, peripheral nerve neurotoxicity and ototoxicity were grade 1 or less.

\section{Discussion}

OPG in NF1 typically becomes symptomatic during the first decade of life $(2,32,33)$. Rarely, as in our case, the presymptomatic latent period may be much longer and last into late adolescence or early adulthood, underscoring the need for appropriate long-term ophthalmological surveillance (18). The clinical and pathological characteristics of such patients is different from the cohorts of adult patients with low-grade glioma treated with adjuvant therapy in recent studies $(29$, 31 ). Therefore, guidance is probably better extrapolated from the pediatric experience with NF1 and OPG. This report demonstrates that standard pediatric non-targeted chemotherapy with carboplatin and VCR is feasible, effective and well-tolerated after dose adjustment in adults with NF1 and symptomatic OPG.

The described case is instructive as it highlights the risk for late and non-malignant progression of OPG in NF1, with a sustained volumetric and functional response to pediatrictype chemotherapy. This suggests that a late manifestation of OPG in NF1 may be treated with extended childhood therapeutic regimen. Data for long-term outcomes and sideeffects from RAS/RAF/MAP/MEK pathway inhibition in
NF1- and non-NF1 OPG will be obtained in future years and will influence our sequential non-surgical treatment paradigms. Until then, it is important to keep in mind that we may resort to the time-tested conventional platin- and vinca-based therapy even in adult NF1-associated OPG.

\section{References}

1 von Recklinghausen F: Über die multiplen Fibrome der Haut und ihrer Beziehung zu den multiplen Neuromen. Hirschwald, Berlin, 1882.

2 Cimino PJ and Gutmann DH: Neurofibromatosis type 1. Handb Clin Neurol 148: 799-811, 2018.

3 Friedman JM, Gutmann DH, MacCollin M and Riccardi VM (eds.): Neurofibromatosis: Phenotype, Natural History, and Pathogenesis. Third Edition. The Johns Hopkins University Press, Baltimore, 1999.

4 Parrozzani R, Clementi M, Frizziero L, Miglionico G, Perrini P, Cavarzeran F, Kotsafti O, Comacchio F, Trevisson E, Convento $\mathrm{E}$, Fusetti $\mathrm{S}$ and Midena E: In vivo detection of choroidal abnormalities related to NF1: Feasibility and comparison with standard NIH diagnostic criteria in pediatric patients. Invest Ophthalmol Vis Sci 56: 6036-6042, 2015.

5 Hudson A: Primary tumors of the optic nerve. R London Ophthalmol Hosp Rep 18: 317-439, 1912.

6 Davis FA: Plexiform neurofibromatosis (von Recklinghausen's disease) of the orbit and globe, with associated glioma of the optic nerve and brain: Report of a case. Trans Am Ophthalmol Soc 37: 250-271, 1939.

7 Davis FA: Primary tumors of the optic nerve (a phenomenon of Recklinghausen's disease). Arch Ophthalmol 23: 735-821, 9571018, 1940

8 Dutton JJ: Gliomas of the anterior visual pathway. Surv Ophthalmol 38: 427-452, 1994.

9 Listernick R, Charrow J, Greenwald M and Mets M: Natural history of optic pathway tumors in children with neurofibromatosis type 1: A longitudinal study. J Pediatr 125: 63-66, 1994.

10 Czyzyk E, Jóźwiak S, Roszkowski M and Schwartz RA: Optic pathway gliomas in children with and without neurofibromatosis 1. J Child Neurol 18: 471-478, 2003.

11 Blazo MA, Lewis RA, Chintagumpala MM, Frazier M, McCluggage $\mathrm{C}$ and Plon SE: Outcomes of systematic screening for optic pathway tumors in children with neurofibromatosis type 1. Am J Med Genet A 127A: 224-229, 2004. 
12 Jahraus CD and Tarbell NJ: Optic pathway gliomas. Pediatr Blood Cancer 46: 586-596, 2006.

13 King A, Listernick R, Charrow J, Piersall L and Gutmann DH: Optic pathway gliomas in neurofibromatosis type 1: The effect of presenting symptoms on outcome. Am J Med Genet A 122A: 95-99, 2003.

14 Campen CJ and Gutmann DH: Optic pathway gliomas in neurofibromatosis type 1. J Child Neurol 33: 73-81, 2018.

15 Listernick R, Louis DN, Packer RJ and Gutmann DH: Optic pathway gliomas in children with neurofibromatosis 1: Consensus statement from the NF1 Optic Pathway Glioma Task Force. Ann Neurol 41: 143-149, 1997.

16 Hochstrasser H, Boltshauser E, and Valavanis A: Brain tumors in children with von Recklinghausen neurofibromatosis. Neurofibromatosis 1: 233-239, 1988.

17 Lund AM and Skovby F: Optic gliomas in children with neurofibromatosis type 1. Eur J Pediatr 150: 835-838, 1991.

18 Friedrich RE and Nuding MA: Optic pathway glioma and cerebral focal abnormal signal intensity in patients with neurofibromatosis type 1: Characteristics, treatment choices and follow-up in 134 affected individuals and a brief review of the literature. Anticancer Res 36: 4095-4121, 2016.

19 Listernick R, Charrow J, Greenwald MJ and Esterly NB: Optic gliomas in children with neurofibromatosis type 1 . J Pediatr 114: 788-792, 1989.

20 Lewis RA, Gerson LP, Axelson KA, Riccardi VM and Whitford RP: von Recklinghausen neurofibromatosis. II: Incidence of optic gliomata. Ophthalmology 91: 929-935, 1984.

21 Listernick R, Ferner RE, Piersall L, Sharif S, Gutmann DH and Charrow J: Late-onset optic pathway tumors in children with neurofibromatosis 1. Neurology 63: 1944-1946, 2004.

22 Ater JL, Xia C, Mazewski CM, Booth TN, Freyer DR, Packer RJ, Sposto R, Vezina G and Pollack IF: Nonrandomized comparison of neurofibromatosis type 1 and nonneurofibromatosis type 1 children who received carboplatin and vincristine for progressive low-grade glioma: A report from the Children's Oncology Group. Cancer 122(12): 1928-1936, 2016.

23 Lassaletta A, Scheinemann K, Zelcer SM, Hukin J, Wilson BA, Jabado N, Carret AS, Lafay-Cousin L, Larouche V, Hawkins CE, Pond GR, Poskitt K, Keene D, Johnston DL, Eisenstat DD, Krishnatry R, Mistry M, Arnoldo A, Ramaswamy V, Huang A, Bartels U, Tabori U and Bouffet E: Phase II weekly vinblastine for chemotherapy-naïve children with progressive low-grade glioma: A Canadian Pediatric Brain Tumor Consortium Study. J Clin Oncol 34(29): 3537-3543, 2016.

24 Hargrave D: PD-013 The ophthalmologic recommendations for standardized visual assessment and treatment indications within the next SIOP-LGG (LOGGIC) trial including children with visual pathway glioma. 48th Congress of the International Society of Paediatric Oncology (SIOP), Dublin, 19. October 2016 - 22. October 2016 http://discovery.ucl.ac.uk/10032505/

25 Lin R, Hug EB, Schaefer RA, Miller DW, Slater JM and Slater JD: Conformal proton radiation therapy of the posterior fossa: A study comparing protons with three-dimensional planned photons in limiting dose to auditory structures. Int J Radiat Oncol Biol Phys 48: 1219-1226, 2000.
26 Erkal HS, Serin M, and Cakmak A: Management of optic pathway and chiasmatic-hypothalamic gliomas in children with radiation therapy. Radiother Oncol 45: 11-15, 1997.

27 Binning MJ, Liu JK, Kestle JR, Brockmeyer DL and Walker ML: Optic pathway gliomas: a review. Neurosurg Focus 23: E2, 2007.

28 Brown PD, Anderson SK, Carrero XW, O'Neill BP, Giannini C, Galanis E, Shah SA, Abrams RA, Curran WJ, Buckner JC and Shaw EG: Adult patients with supratentorial pilocytic astrocytoma: Long-term follow-up of prospective multicenter clinical trial NCCTG-867251 (Alliance). Neurooncol Pract 2(4): 199-204, 2015.

29 Shaw EG, Wang M, Coons SW, Brachman DG, Buckner JC, Stelzer KJ, Barger GR, Brown PD, Gilbert MR and Mehta MP: Randomized trial of radiation therapy plus procarbazine, lomustine, and vincristine chemotherapy for supratentorial adult low-grade glioma: Initial results of RTOG 9802. J Clin Oncol 30(25): 3065-3070, 2012.

30 Wahl M, Phillips JJ, Molinaro AM, Lin Y, Perry A, Haas-Kogan DA, Costello JF, Dayal M, Butowski N, Clarke JL, Prados M, Nelson S, Berger MS and Chang SM: Chemotherapy for adult low-grade gliomas: clinical outcomes by molecular subtype in a phase II study of adjuvant temozolomide. Neurooncology 19(2): 242-251, 2017.

31 Baumert BG, Hegi ME, van den Bent MJ, von Deimling A, Gorlia T, Hoang-Xuan K, Brandes AA, Kantor G, Taphoorn MJ, Hassel MB, Hartmann C, Ryan G, Capper D, Kros JM, Kurscheid S, Wick W, Enting R, Reni M, Thiessen B, Dhermain F, Bromberg JE, Feuvret L, Reijneveld JC, Chinot O, Gijtenbeek JM, Rossiter JP, Dif N, Balana C, Bravo-Marques J, Clement PM, Marosi C, Tzuk-Shina T, Nordal RA, Rees J, Lacombe D, Mason WP and Stupp R: Temozolomide chemotherapy versus radiotherapy in high-risk low-grade glioma (EORTC 2203326033): A randomised, open-label, phase 3 intergroup study. Lancet Oncol 17(11): 1521-1532, 2016.

32 Dalla Via P, Opocher E, Pinello ML, Calderone M, Viscardi E, Clementi M, Battistella PA, Laverda AM, Da Dalt L and Perilongo G: Visual outcome of a cohort of children with neurofibromatosis type 1 and optic pathway glioma followed by a pediatric neurooncology program. Neuro Oncol 9: 430-437, 2007.

33 Kornreich L, Blaser S, Schwarz M, Shuper A, Vishne TH, Cohen IJ, Faingold R, Michovitz S, Koplewitz B and Horev G: Optic pathway glioma: Correlation of imaging findings with the presence of neurofibromatosis. Am J Neuroradiol 22: 1963-1969, 2001.
Received December 13, 2018

Revised December 27, 2018 Accepted January 7, 2019 